\title{
Toxicidade retiniana do gás hexafluoreto de enxofre, líquido perfluorocarbono, ar e solução salina balanceada em olhos de coelhos
}

\author{
Sulfur hexafluoride gas, perfluorocarbon liquid, air and balanced salt solution \\ retinal toxicity in rabbit eyes
}

\author{
Eduardo Vieira de Souza ${ }^{1}$ \\ Teruo Aihara ${ }^{2}$ \\ Nivaldo Vieira de Souza ${ }^{3}$ \\ Joaquim Coutinho Neto ${ }^{4}$
}

Trabalho realizado no Departamento de Oftalmologia, Otorrinolaringologia e Cirurgia de Cabeça e Pescoço da Faculdade de Medicina de Ribeirão Preto (USP).

Pós-graduando, nível Doutorado do Departamento de Oftalmologia, Otorrinolaringologia e Cirurgia de Cabeça e Pescoço da Faculdade de Medicina de Ribeirão Preto da Universidade de São Paulo (USP). Ribeirão Preto (SP). Médico do Setor de Retina do Departamento de Oftalmologia do Hospital Santa Casa de Misericórdia de São Paulo. São Paulo (SP)

Professor Doutor do Departamento de Oftalmologia, Otorrinolaringologia e Cirurgia de Cabeça e Pescoço da Faculdade de Medicina de Ribeirão Preto da USP Ribeirão Preto (SP)

${ }^{4}$ Professor do Departamento de Bioquímica e Imunologia da Faculdade de Medicina de Ribeirão Preto da USP Ribeirão Preto (SP)

Endereço para correspondência: Rua Eliseu Guilherme, 678 - Ribeirão Preto (SP) CEP 14025-020

E-mail: esmvsouza@yahoo.com.br

Recebido para publicação em 11.11.2004

Versão revisada recebida em 15.03.2005

Aprovação em 15.04.2005

\begin{tabular}{|l|}
\hline RESUMO \\
\hline Objetivo: Determinar a toxicidade retiniana do gás hexafluoreto de enxofre, \\
líquido perfluorocarbono e solução salina balanceada em olhos de coe- \\
lhos. Métodos: Foram analisados 22 olhos de 16 coelhos albinos da raça \\
Nova Zelândia divididos em grupos: solução salina balanceada (7 olhos); \\
hexafluoreto de enxofre (4 olhos), líquido perfluorocarbono (5 olhos) e \\
controle (6 olhos). Após aspiração de 0,3 ml de humor vítreo, foi injetado \\
a mesma quantidade de solução salina balanceada ou hexafluoreto de \\
enxofre a 100\% ou líquido perfluorocarbono na cavidade vítrea. O grupo \\
controle não foi submetido a nenhum procedimento. Três semanas depois \\
o humor vítreo foi coletado para análise bioquímica e olho enucleado para \\
análise histológica. Resultados: Os olhos dos animais que receberam \\
injeção de hexafluoreto de enxofre e líquido perfluorocarbono mostraram \\
significativo aumento da concentração vítrea de glutamato quando com- \\
parado aos grupos solução salina balanceada e controle (p<0,05). A \\
análise histológica confirmou os achados bioquímicos mostrando altera- \\
ções como disrupção do segmento externo dos fotorreceptores, afilamento \\
das camadas plexiforme interna e externa, diminuição do número de \\
núcleos na camada ganglionar e nuclear interna, edema e presença de \\
macrófagos nas camadas superficiais. Estas alterações foram mais acen- \\
tuadas no grupo líquido de perfluorocarbono em relação ao grupo hexa- \\
fluoreto de enxofre. Nãoforamobservadas alterações histológicas retinianas \\
significativas nos grupos solução salina balanceada e controle. Conclu- \\
são: A presença de gás hexafluoreto de enxofre e líquido perfluorocarbono \\
na câmara vítrea se mostrou potencialmente tóxica para a retina de coelhos \\
quando comparado ao grupo controle e solução salina balanceada. \\
\hline
\end{tabular}

Descritores: Retina/lesões; Descolamento retiniano/cirurgia; Corpo vítreo/efeito de drogas; Hexafluoreto de enxofre/toxicidade; Líquido perfluorocarbono/toxicidade; Ácido glutâmico/ toxicidade; Coelhos

\section{INTRODUCÃO}

Nos últimos 50 anos, grandes avanços ocorreram para a elucidação da patogênese e tratamento do descolamento de retina (DR), principalmente depois que Schepens ${ }^{(1)}$ desenvolveu e popularizou o uso da oftalmoscopia binocular indireta. Desde então houve uma revolução nas formas de tratamento do DR com o aprimoramento de técnicas já existentes e o aparecimento de outras ${ }^{(2-4)}$. Juntamente com estes incrementos, novas substâncias foram adicionadas ao arsenal cirúrgico do DR. O gás hexafluoreto de enxo- 
fre $\left(\mathrm{SF}_{6}\right)$ constitui um importante recurso na terapêutica do DR, podendo ser utilizado tanto no DR simples, por meio da retinopexia pneumática, como nos DR complexos associados a vitrectomia com traumatismo, proliferação vitreoretiniana, retinopatia diabética, roturas gigantes ou roturas posteriores ${ }^{(5)}$. Devido ao pequeno índice de complicação e ao grande percentual de sucesso cirúrgico, o gás $\mathrm{SF}_{6}$ já está definitivamente incorporado ao arsenal terapêutico do DR.

Os líquidos perfluorocarbonados (LPFC) surgiram em 1982, quando Haidt et al. ${ }^{(6)}$ lançaram a idéia do seu uso como substituto do humor vítreo devido às suas características físicas como alta densidade, baixa viscosidade, transparência óptica, boa tensão superficial e imiscibilidade em água, óleo ou sangue. Todas estas características facilitam a manipulação desta substância pelo cirurgião durante o procedimento, podendo ser usada para aplanar a retina sem a necessidade de retinotomia, estabilizar mecanicamente a retina durante a dissecção de membranas, detecção de áreas de tração, visibilização de membranas sub-retinianas, proteção da retina durante a remoção de fragmentos e localização de roturas retinianas que não puderam ser vistas anteriormente ${ }^{(7)}$. Miyamoto et al. foram os primeiros a realizar estudos com estas substâncias por períodos prolongados dentro do olho ${ }^{(8)}$. Os LPFC se mostraram tóxicos quando em contato prolongado com os olhos de animais, por isso, têm sido usados hoje como substâncias intraoculares temporárias nas cirurgias do segmento posterior, em diferentes condições, e removidas ao final da cirurgia ${ }^{(9-10)}$.

Quando da realização de uma vitrectomia, o humor vítreo removido deve ser reposto de forma temporária ou permanente por alguma substância inerte. Uma das substâncias freqüentemente utilizadas é a solução salina balanceada (BSS). O BSS apresenta características físicas e químicas que o tornam uma das substâncias menos lesivas à retina ${ }^{(11)}$.

Portanto, o BSS, gás SF $_{6}$ e LPFC são substâncias que estão definitivamente incorporadas ao arsenal cirúrgico do oftalmologista ${ }^{(5,7,11)}$. Sua importância e benefícios a oftalmologia são indiscutíveis, entretanto, permanecem ainda algumas dúvidas quanto aos possíveis efeitos lesivos destas substâncias às estruturas oculares, em especial a retina. A busca pela determinação de mecanismos físicos e químicos presentes nestas substâncias que possam lesar o tecido ocular deve ser uma constante. Por isso, o presente estudo tem por objetivo avaliar a toxicidade retiniana do BSS, gás $\mathrm{SF}_{6}$ e LPFC, através do estudo bioquímico e histológico dos olhos de coelhos submetidos à injeção intravítrea destas substâncias.

\section{MÉTODOS}

Foram estudados 22 olhos de 16 coelhos albinos fêmea da raça Nova Zelândia, com peso variando entre 2,0 e $2,5 \mathrm{~kg}$, fornecidos pelo biotério da Faculdade de Medicina de Ribeirão Preto, da Universidade de São Paulo. Todos os animais foram tratados de acordo com os critérios preconizados pela "Association for Research in Vision and Ophthalmology" (ARVO). Os olhos foram divididos em 3 grupos distintos, de acordo com a substância injetada: BSS (7 olhos); $\mathrm{SF}_{6}$ (4 olhos) e LPFC (5 olhos). Seis olhos adelfos escolhidos ao acaso formaram o grupo controle, dois do grupo $\mathrm{SF}_{6}$, dois do grupo LPFC e dois do grupo BSS.

A indução anestésica se fez com uma injeção intramuscular de ketamina (50 mg/kg peso) e xilazina $2 \%(10 \mathrm{mg} / \mathrm{kg}$ peso $)$ e a manutenção com thiopentobarbital sódico $2,5 \%$ na dose de $5 \mathrm{mg} / \mathrm{kg}$ peso via endovenosa. Após anestesia local com instilação de colírio anestésico de cloridato de proximetacaína $0,5 \%$ iniciou-se o procedimento com a colocação do blefarostato e abertura conjuntival de aproximadamente $0,5 \mathrm{~cm}$, às 11 horas, seguida da passagem de um ponto escleral em "U" e esclerotomia com agulha calibre 10 a 2,0 $\mathrm{mm}$ do limbo, introduzida até a porção central do corpo vítreo. Em seguida o humor vítreo foi aspirado $(0,3 \mathrm{ml})$ e pela mesma agulha injetou-se $0,3 \mathrm{ml}$ da substância (BSS, $\mathrm{SF}_{6}$ puro ou LPFC). A perfuração foi selada amarrando-se o ponto escleral, a conjuntiva fechada com injeção subconjuntival de soro fisiológico e instilada uma gota de colírio de tobramicina e dexametasona. Os olhos controle não foram submetidos a qualquer procedimento.

Após três semanas do experimento, sob anestesia, 1,0 ml do humor vítreo de cada olho foi aspirado com uma agulha calibre 10, montado em uma seringa estéril e encaminhado para estudo bioquímico (Figura 1). A análise quantitativa dos aminoácidos presentes no humor vítreo (aspartato, glutamato, serina, glicina, tirosina e lisina) dos coelhos foi feita através da cromatografia líquida de alto desempenho (HPLC). Antes do sacrifício os olhos foram enucleados e fixados em solução tamponada de formaldeído a $10 \%$ para em seguida serem processados para análise histológica da retina que se fez pela microscopia óptica com coloração de hematoxilina-eosina. Utilizou-se um microscópio Zeiss, com lâmpada halogenada e filme "Ektachrome" ASA 64 e ASA 100 para fotos em papel.

A média \pm desvio padrão da concentração vítrea dos aminoácidos estudados (aspartato, glutamato, serina, glicina, tirosina e lisina) foram registrados no programa Origin 6.0 e utilizou-se o teste paramétrico $t$ de Student para análise estatística. O nível de significância foi firmado em $5 \%(\mathrm{p}<0,05)$.

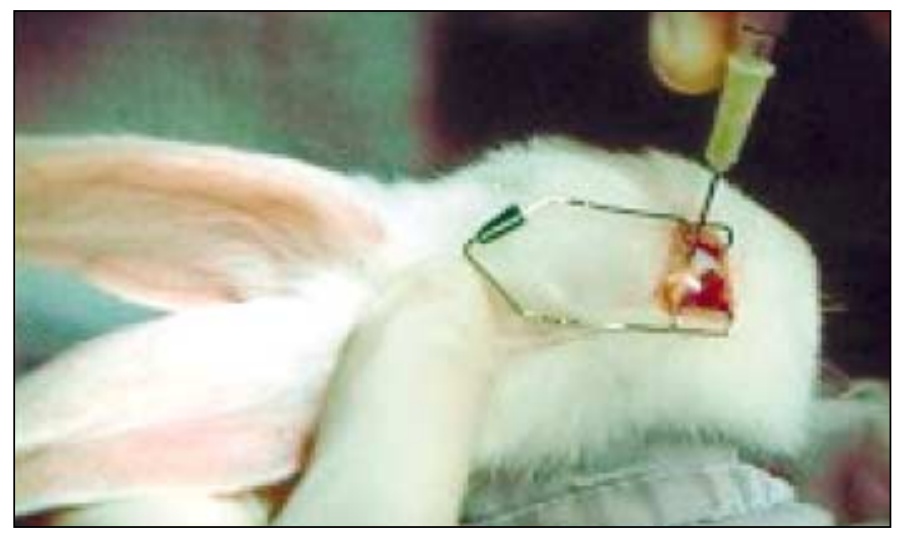

Figura 1 - Fotografia do procedimento de aspiração da amostra vítrea do olho direito do coelho antes da enucleação e sacrifício 


\section{RESULTADOS}

A análise quantitativa do aminoácido excitatório glutamato no humor vítreo de coelhos submetidos à injeção de $\mathrm{SF}_{6}$ e LPFC se mostrou mais elevada em média do que nos olhos submetidos à injeção de BSS e olhos controle. Esta diferença foi estatisticamente significante, como demonstrado na tabela $1 . \mathrm{O}$ gráfico 1 apresenta a distribuição dos outros aminoácidos analisados, mostrando não haver grandes diferenças entre as amostras.

Histologicamente as retinas dos grupos $\mathrm{SF}_{6}$ e LPFC apresentaram intensa vacuolização da camada ganglionar, com picnose, edema e diminuição do número de núcleos. A camada nuclear interna apresentou-se edemaciada, achado este evidenciado pelo aumento da distância entre as camadas ganglionar e nuclear externa, com diminuição do número de núcleos. As camadas mais superficiais mostraram a presença de macrófagos. Alterações foram observadas também nas camadas mais externas como disrupção dos segmentos externos dos fotorreceptores e afilamento da camada plexiforme externa. No grupo LPFC estas lesões se apresentaram de forma mais acentuada, enquanto que no grupo $\mathrm{SF}_{6}$ foram mais brandas.

Todos os grupos (BSS, $\mathrm{SF}_{6}$ e LPFC) apresentaram graus variáveis de vacuolização em diversas camadas da retina (plexiforme interna, ganglionar e nuclear externa). O grupo controle também apresentou vacuolizações só que em menor número.

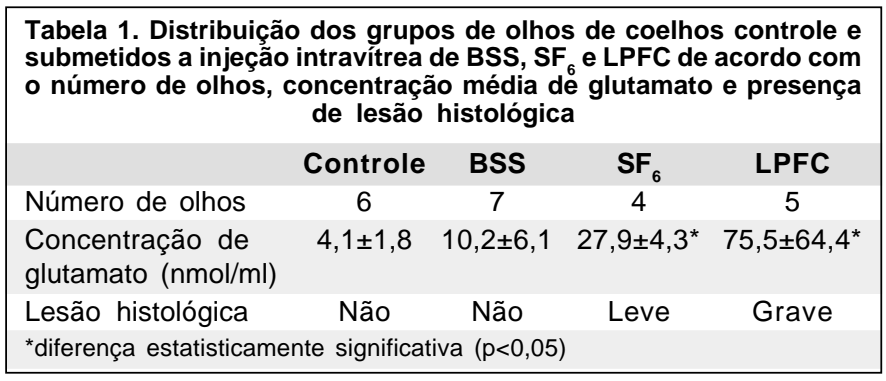

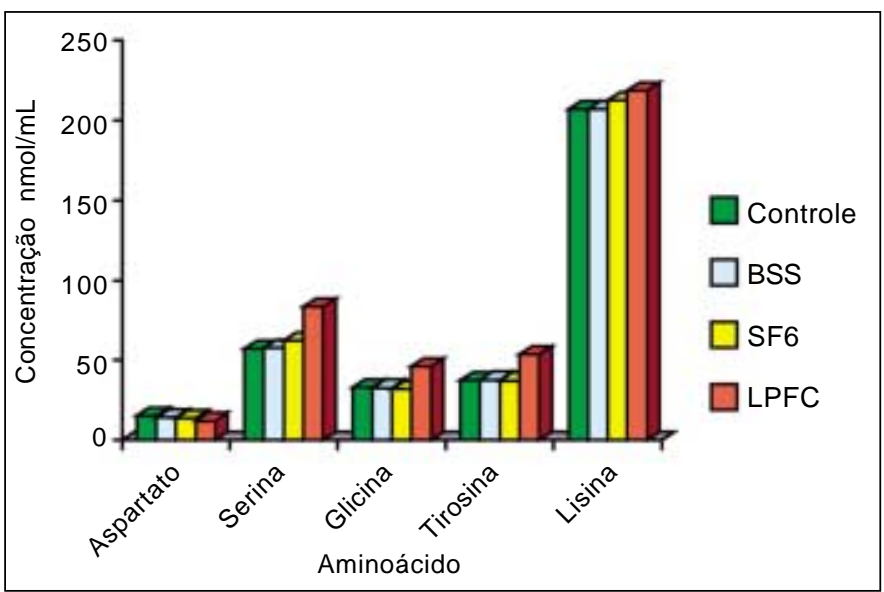

Gráfico 1 - Concentração de aminoácidos do vítreo de coelhos controle e tratados com injeção intravítrea de BSS, SF $_{6}$ e LPFC

\section{DISCUSSÃO}

$\mathrm{O}$ presente estudo demonstrou que o gás $\mathrm{SF}_{6} \mathrm{e}$ o LPFC parecem induzir lesão retiniana quando em contato prolongado com o olho de coelhos. Vários outros estudos experimentais já haviam demonstrado a intolerância do olho ao $\operatorname{LPFC}^{(8,10-11)}$. Alguns autores demonstraram que 6 dias após a injeção intravítrea de LPFC os olhos de coelhos já apresentavam alterações histológicas à microscopia óptica e eletrônica ${ }^{(12)}$. Já o gás $\mathrm{SF}_{6}$, por outro lado, parece não ter propriedades farmacológicas, químicas ou físicas tóxicas à retina ${ }^{(13)}$.

Todos estes estudos foram feitos baseados na histopatologia, não correlacionando a lesão histológica da retina às alterações bioquímicas do humor vítreo. A análise bioquímica do humor vítreo através da quantificação dos aminoácidos excitatórios feita no presente estudo baseou-se nos princípios de Rothman, Olney ${ }^{(14)}$ que, em 1986, postularam a teoria da toxicidade excitatória (neurotoxicidade) apontando os aminoácidos excitatórios, principalmente o glutamato, como um importante agente causal da morte neuronal. A ocorrência de aumento anormal na liberação do glutamato "in vivo" foi demonstrada pela primeira vez por alguns autores, em ratos ${ }^{(15)}$. Acredita-se que este aumento nos níveis de glutamato seja proveniente da liberação extracelular deste aminoácido neurotransmissor, presente nos neurônios e células gliais. A causa deste aumento poderia estar relacionado à falência do sistema de transporte deste aminoácido para o interior das células, às situações em que a morte celular (neurônios e glia) está aumentada ou à ocorrência de falência energética por hipóxia ou anóxia ou em casos de isquemia ${ }^{(14)}$.

Nenhum outro estudo havia demonstrado a mudança da concentração vítrea do glutamato em olhos tratados com $\mathrm{SF}_{6} \mathrm{e}$ LPFC. O aumento da concentração do glutamato no humor vítreo dos animais tratados com $\mathrm{SF}_{6}$ e LPFC, atingindo concentrações médias da ordem de $27,9 \mathrm{nmol} / \mathrm{mL}$ e $75,5 \mathrm{nmol} / \mathrm{mL}$, respectivamente, são altamente tóxicas à retina e, por si, explicariam as lesões histológicas encontradas, ou seja, induziriam a um quadro semelhante à isquemia retiniana. As substâncias $\mathrm{SF}_{6}$ e LPFC poderiam estar agindo nos neurônios retinianos ou células gliais, através de qualquer um dos mecanismos acima mencionados. Estes valores foram significativamente maiores do que as encontradas nos grupos BSS e controle, que apresentaram concentrações de $9,5 \mathrm{nmol} / \mathrm{mL}$ e $4,1 \mathrm{nmol} / \mathrm{mL}$, respectivamente. Resultados semelhantes aos encontrados no presente estudo foram descritos por vários autores ${ }^{(16)}$, que constataram aumento específico da liberação do glutamato e ortofosfoserina (OPS) em retinas de coelhos submetidos à isquemia.

Corroborando com os achados bioquímicos, a análise histológica das retinas tratadas com LPFC mostrou alterações semelhantes às encontradas em outros estudos ${ }^{(8,10)}$. O mecanismo pelo qual a lesão se dá permanece desconhecido, sendo aventadas as possibilidades da toxicidade retiniana da substância e do seu efeito mecânico sobre a retina ${ }^{(17-18)}$. 
De qualquer forma, o aumento significativo da concentração de glutamato no humor vítreo e as alterações histológicas da retina de coelhos tratados com injeção intravítrea de LPFC confirmam a propriedade lesiva desta substância quando em contato prolongado com a retina de coelhos.

$\mathrm{O}$ grupo tratado com $\mathrm{SF}_{6}$ também apresentou alterações histológicas da retina, só que bem menos acentuadas. De forma semelhante, outros autores ${ }^{(19)}$ encontraram alterações histológicas na retina superior de coelhos submetidos à injeção intravítrea de $\mathrm{SF}_{6}$. Entretanto, estes autores consideraram que estas alterações não se mostraram significativamente diferentes das alterações presentes no grupo controle. Apesar de não termos encontrado alterações significativas no grupo controle e BSS, nossa análise histológica mostrou que algumas alterações do grupo $\mathrm{SF}_{6}$ poderiam ser transitórias, corroborando com as conclusões de Doi et al. ${ }^{(19)}$. A presença de vacuolização nas camadas mais internas da retina foi descrita por alguns autores como artefatos do processo de fixação da retina ${ }^{(20)}$, enquanto outros acreditam que sejam decorrentes da quebra da barreira hemato-retiniana ${ }^{(13)}$. De qualquer forma, estas alterações histológicas, podem se tornar importantes devido à presença de altas concentrações de glutamato no humor vítreo.

A utilização de drogas potencialmente neuroprotetoras parece ser uma boa solução na tentativa de reduzir a lesão mediada pelo aumento da concentração de glutamato. Isso pode ser feito através da utilização de drogas que ajam em uma ou mais etapas da cascata que leva ao aumento extracelular de glutamato. Algumas delas já vem sendo estudadas há algum tempo, como é o caso dos redutores de radicais livres ${ }^{(21)}$, substâncias que agem no receptor de glutamato $^{(22)}$, antagonistas dos receptores opióides ${ }^{(23)}$, agentes dopaminérgicos ${ }^{(24)}$, agentes adrenosinérgi$\cos ^{(25)}$ ou antagonistas do cálcio ${ }^{(26)}$. A possibilidade de proteção neuro-retiniana quando da utilização de substitutos do humor vítreo já é alvo de investigações pelos autores.

\section{CONCLUSÃO}

A presença de altas concentrações vítreas de glutamato, juntamente com alterações histológicas da retina de coelhos submetidos à injeção intravítrea de $\mathrm{SF}_{6}$ e LPFC, quando comparado aos grupos controle e BSS, indicam lesão do tecido retiniano.

\section{ABSTRACT}

Purpose: To determine retinal toxicity after intravitreous balanced salt solution, sulfur hexafluoride gas, and perfluorocarbon liquid injection in rabbit eyes. Methods: Twenty-two eyes of sixteen New Zealand albino rabbits were divided into groups: balanced salt solution (7 eyes); sulfur hexafluoride gas (4 eyes); and perfluorocarbon liquid (5 eyes). After the introduction of a needle through the sclera the vitreous was aspirated $(0.3 \mathrm{ml})$, balanced salt solution, sulfur hexafluoride gas $100 \%$ and perfluorocarbon liquid $(0.3 \mathrm{ml})$ were injected into rabbit vitreous cavity. The control group (6 eyes) was not submitted to any procedure. After three weeks the vitreous was aspirated and submitted to biochemical analysis and the eyes prepared for histological analysis. Results: The eyes submitted to perfluorocarbon liquid and sulfur hexafluoride gas injection showed a greater L-glutamate increase in the vitreous compared to balanced salt solution and control groups $(\mathrm{p}<0.05)$. Histological results confirmed small changes in the sulfur hexafluoride group and important lesions in perfluorocarbon liquid group, such as external photoreceptor segment disruption, external and internal plexiform layer thinning, ganglionar and internal nuclear layer decrease of nucleus number, edema, and presence of macrophages in the superficial layers. No major histological changes were observed with balanced salt solution and in control groups. Conclusion: Liquid intravitreous injection of sulfur hexafluoride gas and perfluorocarbon are potentially toxic to rabbit retina, compared to control and balanced salt solution groups.

Keywords: Retina/injuries; Retinal detachment/surgery; Vitreous body/drugs effects; Sulfur hexafluoride/toxicity; Perfluorocarbon liquid/toxicity; Glutamic acid/toxicity; Rabbits

\section{REFERÊNCIAS}

1. Schepens CL. A new ophthalmoscope demonstration. Trans Am Acad Ophthalmol Otolaryngol. 1947;51:298.

2. Machemer R, Buettner H, Norton EW, Parel JM. Vitrectomy: a pars plana approach. Trans Am Acad Ophthalmol Otolaryngol. 1971;75(4):813-20.

3. Hilton GF, Grizzard WS. Pneumatic retinopexy. A two step outpatient operation without conjunctival incision. Ophthalmology. 1986;93(5):626-41.

4. Lincoff HA, Baras I, McLean J. Modifications to the Custodis procedures for retinal detachment. Arch Ophthalmol. 1965;73:160-3.

5. Nehemy MB. Gases em cirurgia vitreoretínica. Arq Bras Oftalmol. 1996; 59(1):969.

6. Haidt SJ, Clark Junior LC, Ginberg J. Liquid perfluorocarbon replacement of the eye. Invest Ophthalmol Vis Sci. 1982;22(Suppl):233-9.

7. Peyman GA, Schulman JA, Sullivan B. Perfluorocarbon liquids in ophthalmology. Surv Ophthalmol. 1995;39(5):375-95.

8. Miyamoto K, Refojo MF, Tolentino FI, Fournier GA, Albert DM. Perfuoroether liquid as a long-term vitreous substitute. An experimental study. Retina 1984;4(4):264-8.

9. Moreira Jr CA, Liggett P, Moreira H. Utilização de um novo perfluoroquímico líquido na cirurgia vítreo-retiniana: estudo experimental. Arq Bras Oftalmol. 1992;55(1):63-9.

10. Queiroz JM Jr, Blanks JC, Özler AS, Alfaro DV, Liggett PE. Subretinal perfluorocarbon liquids. An experimental study. Retina. 1992;12(3 Suppl):S33-9.

11. Saornil Alvarez MA, Pastor Jimeno JC. Role of the intraocular irrigating solution in the pathogenesis of postvitrectomy retinal edema. Curr Eye Res. 1987:6(12):1369-79.

12. Eckardt C, Nicolai U, Winter M, Knop E. Experimental intraocular tolerance to liquid perfluoroctane and perfluoropolyether. Retina. 1991;11(4):375-84.

13. Juzoji H, Iwasaki T, Usui M, Hasemi M, Yamakawa N. Histological study of intraocular changes in rabbits after intravitreal gas injection. Jpn J Ophthalmol. 1997;41(5):278-83.

14. Rothman SM, Olney JW. Glutamate and the pathophysiology of hypoxicischemic brain damage. Ann Neurol. 1986;19(2):105-11.

15. Coutinho-Netto J, Abdul-Ghani AS, Collins JF, Bradford HF. Is the glutamate a trigger factor in epileptic hyperactivity? Epilepsia. 1981;22(3):289-96.

16. Louzada-Junior P, Dias JJ, Santos WF, Lachat JJ, Bradford HF, CoutinhoNetto J. Glutamate release in experimental ischemia of the retina: an approach using microdialysis. J Neurochem. 1992;59(1):358-63. 
17. Velikay M, Wedrich A, Stolba U, Datlinger P, Li Y, Binder S. Experimental long-term vitreous replacement with purified and non purified Perfluorodecaline. Am J Ophthalmol. 1993;116(5):565-70.

18. Velikay M, Stolba U, Wedrich A, Li Y, Datlinger P, Binder S. The effect of chemical stability and purification of perfluorocarbon liquids in experimental extended-term vitreous substitution. Graefes Arch Clin Exp Ophthalmol. 1995;233(1):26-30.

19. Doi M, Ning M, Semba R, Uji Y, Refojo MF. Histopathologic abnormalities in rabbit retina after intravitreous injection of expansive gases and air. Retina. 2000;20(5):506-13.

20. Doi M, Refojo MF. Histopathology of rabbit eyes with intra-vitreous siliconefluorosilicone copolymer oil. Exp Eye Res. 1994;59(6):737-46.

21. Szabo ME, Droy-Lefaix MT, Doly M, Carre C, Braquet P. Ischemia and reperfusion-induced histologic changes in the rat retina. Demonstration of freeradical mediated mechanism. Invest Ophthalmol Vis Sci. 1991;32(5):1471-8.

22. Yoon TH, Marmor MF. Dextromethorphan protects retina against ischemic injury in vivo. Arch Ophthalmol. 1989;107(3):409-11.

23. Lam TT, Takahashi K, Tso MO. The effect of naloxone on retina ischaemia in rats. J Ocular Pharmacol. 1994;10(2):481-92.

24. Kashii S, Takahashi M, Mandai M, Shimizu H, Honda Y, Sasa M, et al. Protective action of dopamine against glutamate neurotoxicity in the retina. Invest Ophthalmol Vis Sci. 1994;35(2):685-95.

25. Larsen A, Osborne NN. Adenosine is a neuroprotective agent in retinal ischaemia. Vision Res. 1995;35(Suppl 340):S214.

26. Takahashi K, Lam TT, Edward DP, Bichi ER, Tso MO. Protective effects of flunarizine on ischaemic injury in the rat retina. Arch Ophthalmol. 1992;110:862-70.

\section{Agora seus artigos científicos}

para publicação nos Arquivos Brasileiros

de Oftalmologia devem ser submetidos por

via eletrônica.

Saiba mais sobre esta ferramenta

fácil e moderna de gerenciamento

editorial dos ABO.

Visite o site eletrônico dos ABO

ww w. aboonline.com.br 